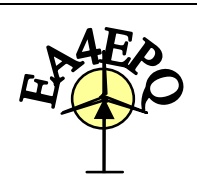

International Conference on Renewable Energies and Power Quality (ICREPQ'09)

Valencia (Spain), 15th to 17th April, 2009

\title{
Millman's Theorem revisited
}

\author{
G. Aguirre-Zamalloa, F. Uriondo, J.R. Hernández \\ Department of Electrical Engineering \\ Faculty of Engineering \\ University of the Basque Country \\ Alameda Urquijo s/n - 48013 Bilbao (Spain) \\ Phone/Fax number:+0034 946012 000, e-mail: gabriel.aguirre@ehu.es, felipe.uriondo@ehu.es, \\ joseramon.hernandez@ehu.es
}

\begin{abstract}
In this work we present some results bearing on Millman's Network Theorem (MNT for short) with a specific focus towards its application to three phase power systems. First we present an original, insightful proof of the Theorem that simultaneously provides the foundation for the Universal Single Phase Equivalent Circuit (USPEC) framework. Next we introduce two modifications that generalise the standard MNT and render it better adapted to the analysis of three-phase power circuits. The first result is a vectorised version based on the USPEC method which allows a very concise calculation of load voltages and the second is aimed at the calculation of neutral point voltage unbalances in more general power circuits. We illustrate the practical application of these results with the help of several commented examples and besides we provide the Matlab/Octave scripts used to perform the analyses.
\end{abstract}

\section{Key words}

Millman's Theorem, Single-phase Equivalent Circuit, Matrix methods, Voltage Unbalance, Octave/Matlab.

\section{Introduction}

Millman's Network Theorem (onwards MNT for short) is usually presented in electrical engineering textbooks [1] and in standard references [2] as a rule for the association of either real (i.e. lossy) voltage sources in parallel or real current sources in series. For example, in Figure 1 we show the typical circuit layout where the application of MNT produces directly the $\underline{U}_{A B}$ voltage as a function of the circuit parameters $\left\{\underline{E}_{k}\right\}$ and $\left\{\underline{y}_{k}=\underline{z}_{k}^{-1}\right\}$ with $1 \leq k \leq N[3]:$

$$
\underline{U}_{A B}=\left(\sum_{k=1}^{N} \underline{y}_{k}\right)^{-1} \cdot\left(\sum_{k=1}^{N} \underline{y}_{k} \underline{E}_{k}\right)
$$

To begin with we present in Section 2 a simple, new circuit theoretical proof that establishes MNT and simultaneously rewards us with the key equation of the Universal Single Phase Equivalent Circuit (USPEC) framework [4] that we will be using extensively in this work. We briefly summarise the basics of the method and additionally we provide a fully commented example programmed in the open environment Octave (a faithful and free Matlab clone) [5] to illustrate the calculation procedures.

In Section 3 we present a straightforward but genuine matrix generalisation of MNT. This result, which reinterprets the circuit of Figure 1 in a fundamental way, features a vectorised form of the theorem and has been obtained with the USPEC method [4].

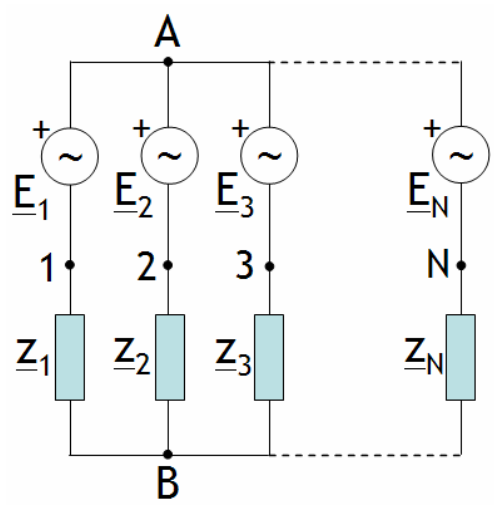

Figure 1: Parallel association of real voltage sources

In Section 4 we discuss a further generalisation of MNT, necessary to apply the theorem to circuits with more complex topologies. The main objective addressed by this research is the study of neutral point unbalances and we have approached this problem using basic circuit theoretical transformations that preserve the electrical equivalence of the circuits and that allow to recast them in simple, standardised forms. Next, in section 5 we illustrate the gist of these results by applying them, with great effect, to the analysis of two simple but cogent examples. Finally come the conclusions of the work and in the Appendix we comment on one numerical 
implementation of the method in the Matlab/Octave environment and describe some coded examples.

\section{Simple proof of MNT}

On purpose and without loss of generality we take $N=4$, see Figure 2. This circuit has 2 nodes and 4 branches so that 8 independent equations can be found: Kirchhoff's laws provide 4 (KCL: 1 \& KVL: 3) plus 4 branch equations. Of these only 4 are nontrivial which in matrix form read:

$$
\left(\begin{array}{ccc:c}
\underline{z}_{1} & 0 & 0 & 1 \\
0 & \underline{z}_{2} & 0 & 1 \\
0 & 0 & \underline{z}_{3} & 1 \\
\hdashline 1 & 1 & 1 & -\underline{y}_{N}
\end{array}\right) \cdot\left(\begin{array}{l}
\underline{I}_{1} \\
\underline{I}_{2} \\
\underline{I}_{3} \\
\hdashline \underline{U}
\end{array}\right)=\left(\begin{array}{l}
\underline{E}_{1} \\
\underline{E}_{2} \\
\underline{E_{3}} \\
\hdashline 0
\end{array}\right)
$$

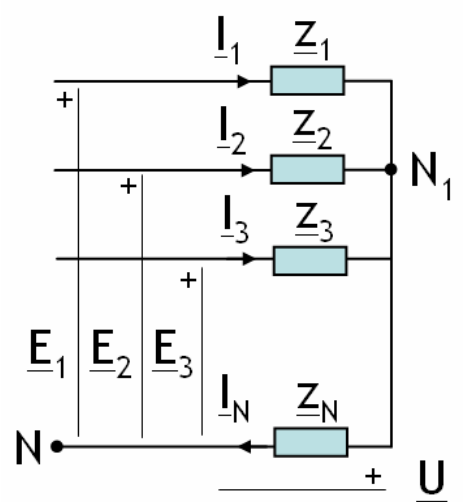

Figure 2: Elementary three phase circuit

All what circuit theory has to say about the circuit of Figure 2 (onwards Circuit 2, for short) is contained in this MNA like matrix equation [6]. These partitioned matrices allow for block reduction [7] so as to eliminate one or several variables from the system. Clearly, if we eliminate $\mathrm{I} \equiv\left(\begin{array}{lll}\underline{I}_{1} & \underline{I}_{2} & \underline{\mathrm{I}}_{3}\end{array}\right)^{\mathrm{T}}$ we get $\underline{\mathrm{U}}$ which is again exactly given by (1)!. Else, if (with the proviso that $\underline{y}_{\mathrm{N}}$ be not zero) we eliminate $\underline{U}$ we get the "single-phase" equation $\mathrm{E}=\mathrm{Z} \cdot \mathrm{I}$ where $\mathrm{E} \equiv\left(\begin{array}{lll}\underline{E}_{1} & \underline{\mathrm{E}}_{2} & \underline{\mathrm{E}}_{3}\end{array}\right)^{\mathrm{T}}$, and

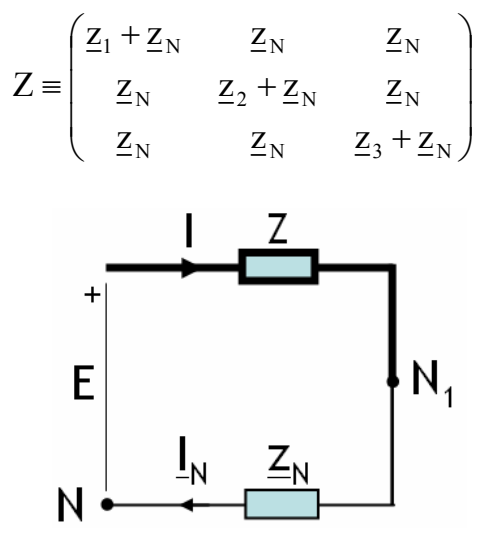

Figure 3: USPEC equivalent of circuit 2

This matrix single-phase equation is the basis of the USPEC method which essentially boils down to consistently applying this result to all three-phase power circuits: open lines receive "big enough" $\underline{z}_{N}$ impedances and delta connected loads are replaced with equivalent star connected loads via Kennelly's Theorem [1][3]. We realise that somehow USPEC \& MNT are independent and complementary methods as each reflect only part of the full equation system (2).

As shown in Figure 3 we often depict only the next-tolast USPEC reduced circuit (the "USPEC" equivalent) in order to keep the neutral points apparent and to distinctly display the multiplexed phase signals (wider lines) and the neutral returns. Obviously in this framework current and voltage variables are vectors and immitances are matrices so that matrix multiplication and inversion are implied and the order of factors matters.

\section{Matrix generalisation of MNT}

In order to illustrate the extraordinary tidiness of the vector method, we propose to analyze the simple threephase power circuit displayed in Figure 4. In this circuit where a load is fed from two real voltage sources, we count 7 nodes and 12 branches which amount to 12 MNA like equations and unknowns. Still, if we resort to either nodal or mesh methods, we have to write 6 equations for 6 unknowns (or equivalently fill a $12 \times 6$ incidence matrix). In contrast, the USPEC equivalent shown in Figure 5 is wonderfully simpler: 2 nodes, 3 branches for 3 MNA equations which bear 2 mesh-like equations or, remarkably, a single node-like equation which is none other than the MNT equation again, in matrix disguise:

$$
U=\left(\begin{array}{l}
\underline{U}_{R O} \\
\underline{U}_{S O} \\
\underline{U}_{T O}
\end{array}\right)=\left(Y_{1}+Y_{2}+Y\right)^{-1} \cdot\left(Y_{1} E_{1}+Y_{2} E_{2}\right)
$$

where $Y \equiv Z^{-1}, Z$ being given by (3). Notice that while the voltages-across-loads $U$ vector is readily calculated, the neutral point unbalances are not directly reachable; again, this fact reflects the previously mentioned complementary status of the two methods.

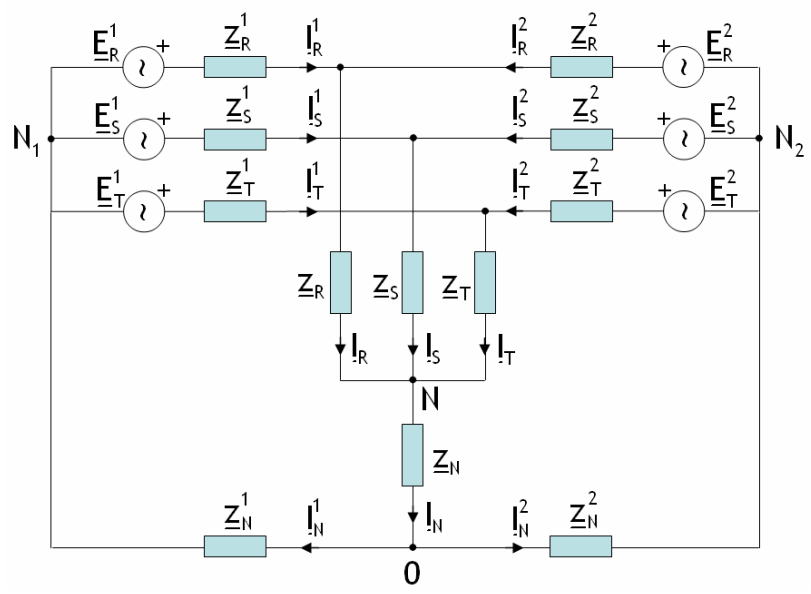

Figure 4: A more complex three phase circuit 
It is very noteworthy how nicely equation (4) subsumes equation (1) for the case $N=3$. As indicated, a fully commented numerical example is presented in the Appendix.

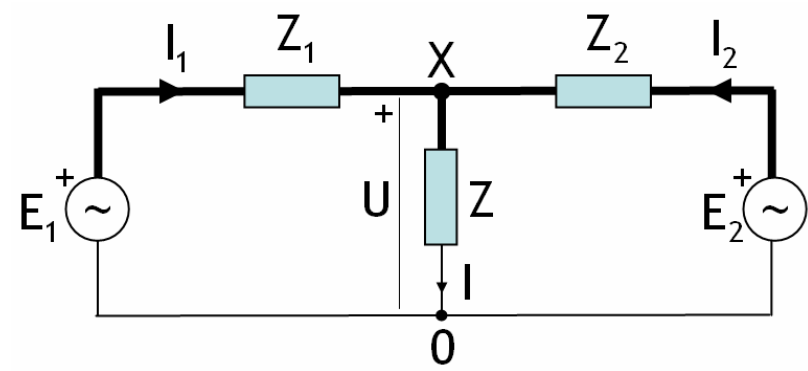

Figure 5: USPEC equivalent of Circuit 4. Here $X=\{R, S, T\}$.

As an interesting aside we want to comment on two recursive features inherent to these results. First, the structure of both the vector (4) and the scalar (1) MNT equations comply with the recursive application of the pairing expressions (at the $\mathrm{N}^{\text {th }}$ step):

$$
\left\{\begin{array}{l}
(a) \quad Y_{\text {old }} \rightarrow Y_{\text {new }}=Y_{\text {old }}+Y_{N} \\
\text { (b) } E_{\text {old }} \rightarrow E_{\text {new }}=Y_{\text {new }}^{-1} \cdot\left(Y_{\text {old }} \cdot E_{\text {old }}+Y_{N} \cdot E_{N}\right)
\end{array}\right.
$$

because, if we know how to combine two real voltage sources then by "complete induction" [8] we know how to combine $\mathrm{N}$ of them.

Second, the USPEC reduction can be pushed further. Let us take, for the sake of the argument, a three-phase load fed from three different three-phase sources. As already explained its USPEC-reduced circuit looks like that of Figure 2. Now, by further USPEC-reduction of this USPECreduced circuit, the original circuit gets equivalently represented by a fictitious single-phase circuit whose elements involve $9 \times 9$ matrices. A numerical example is presented in the Appendix.

\section{Application of MNT to more general circuits}

Equation (1) is extremely valuable and is often used in everyday practice, but does not exhaust the full contents of the theorem. As it stands, full-fledged Millman's Theorem [3] is a result stronger than what is implied by (1) since, quite remarkably, it applies to the much more general situation depicted in Figure 6. In this circuit A stands for an arbitrary point (either inside or outside the box) and MNT establishes an equation which calculates the voltage $\underline{U}_{A B}$ as a function of the circuit parameters $\left\{\underline{U}_{A k}\right\}$ and $\left\{\underline{z}_{k}\right\}$ where in general $1 \leq k \leq N$.

However, this most general circuit is far too abstract to be of any practical interest. On this account we have worked out a series of circuits of intermediate complexity specifically suited for the analysis of three-phase power systems that warrant the application of MNT to practical real circuits.

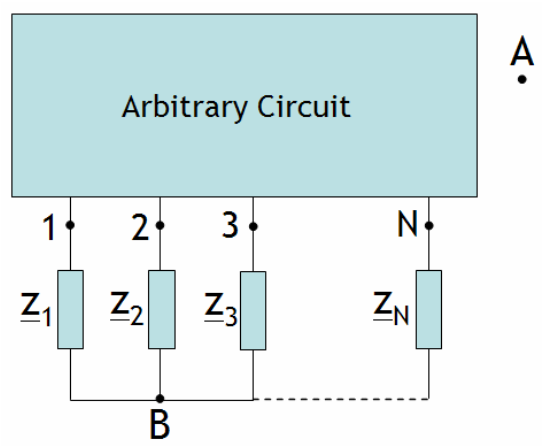

Figure 6: Most general circuit to apply Millman to

An example of such a circuit is Circuit 4, of course. Let us assume for instance that we are interested in calculating the neutral-to-neutral voltage unbalance $\underline{U}_{N N_{1}}$. The first step of the analysis consists in applying the substitution theorem [3], which is a clever circuit theoretical trick not to be confused with "equivalence": substitution provides a "local" equivalent valid only for the specific working point at hand. The circuit so transformed is depicted in Figure 7. Noticeably, the tagged current sources are used to represent any realizable load state of the right-hand branches of the circuit. As a result, these sources are not constant but, on the contrary, depend functionally on all the elements of the original circuit. Clearly this circuit reduces essentially to that of Figure 2 when $\underline{J}_{k}^{2 \#}=0 \quad(k=R, S, T)$.

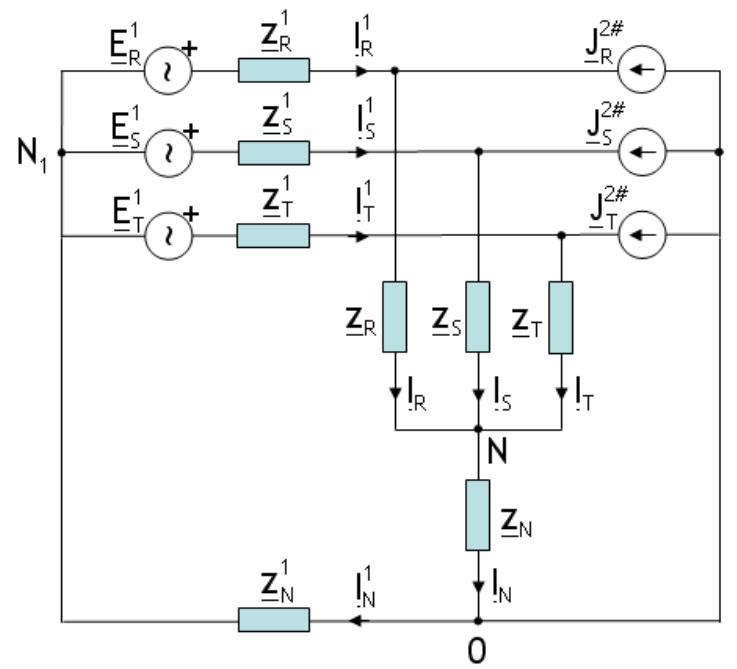

Figure 7: Transformation of Circuit 4 in order to calculate $\underline{\mathrm{U}}_{\mathrm{NN} 1}$

The analysis proceeds by further applying one or several more equivalence transformations: current source "motion" [3], Thevenin's theorem [9] to change real current sources into real voltage sources, USPECreduction, etc. In Figure 8 below we show the very first step of the application of current source motion. Finally, it comes out that the transformed circuit develops into the original Circuit 1 (taking $N=4$ ) with the proviso that $A \equiv N, B \equiv N_{1}$ and the correspondences indicated in Table 1 be applied (hereafter we drop the '\#' tags). 


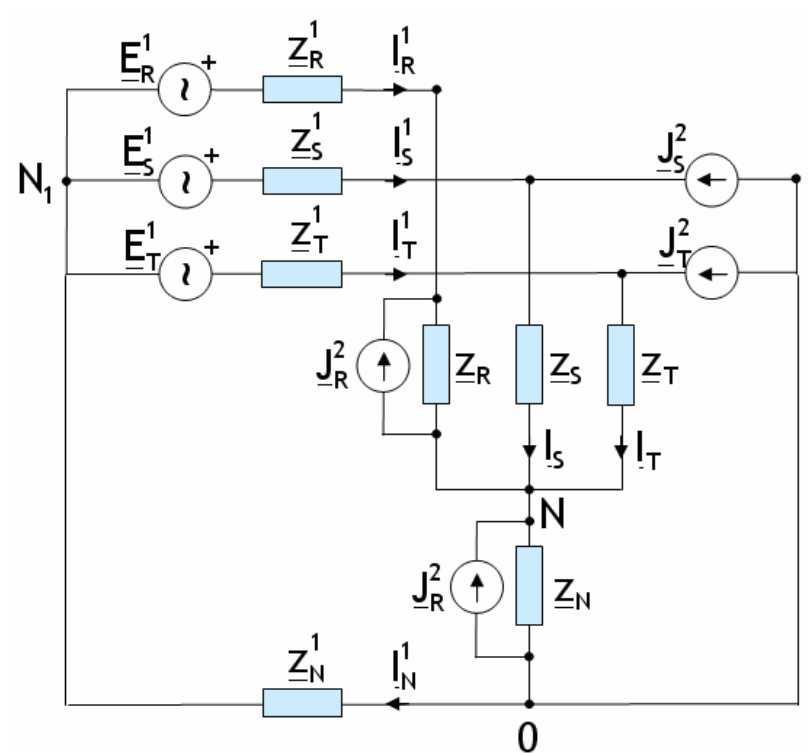

Figure 8: example of current source motion applied to Circuit 7

Table 1: Correspondences between Circuits 7 and $1(k=\mathrm{R}, \mathrm{S}, \mathrm{T})$

\begin{tabular}{|ccc|}
\hline Figure 7 & $\rightarrow$ & Figure 1 \\
$\underline{E}_{k}^{1}-\underline{z}_{k} \cdot \underline{J}_{k}^{2}$ & $\rightarrow$ & $\underline{E}_{k}$ \\
$\underline{z}_{N} \cdot \underline{J}_{0}^{2}$ & $\rightarrow$ & $\underline{E}_{N}$ \\
$\underline{z}_{k}^{1}+\underline{z}_{k}$ & $\rightarrow$ & $\underline{z}_{k}$ \\
$\underline{z}_{N}^{1}+\underline{z}_{N}$ & $\rightarrow$ & $\underline{z}_{N}$ \\
\hline
\end{tabular}

For notational convenience we have defined $\underline{\psi}_{0} \equiv \underline{\psi}_{R}+\underline{\psi}_{S}+\underline{\psi}_{T}$, which save for a 3 factor is the homopolar symmetrical component [3][10] of $\left\{\underline{\psi}_{R}, \underline{\psi}_{S}, \underline{\psi}_{T}\right\}$. Now, by direct application of (1) we get:

$$
\underline{U}_{N N_{1}}=\frac{\sum_{\{3\}}\left(\frac{\underline{E}_{k}^{1}-\underline{z}_{k} \underline{J}_{k}^{2}}{\underline{z}_{k}+\underline{z}_{k}^{1}+\underline{z}_{N} \underline{J}_{k}^{2}} \underline{\underline{z}}_{N}^{1}\right)}{\sum_{\{4\}} \frac{1}{\underline{z}_{k}+\underline{z}_{k}^{1}}}
$$

Where $\{3\}$ and $\{4\}$ stand for summations over $k=R, S, T$ and $k=R, S, T, N$, respectively.

Clearly if the properties $z^{1}-S Y M$ and $z-S Y M$ hold (namely, if the impedances $\{z\}$ and $\left\{z^{1}\right\}$ are symmetric) then,

$$
\underline{U}_{N N_{1}} \propto \frac{\underline{E}_{0}^{1}}{\underline{z}+\underline{z}^{1}}+\underline{J}_{0}^{2} \cdot\left(\frac{\underline{z}_{N}}{\underline{z}_{N}+\underline{z}_{N}^{1}}-\frac{\underline{z}}{\underline{z}+\underline{z}^{1}}\right)
$$

That is to say, if additionally we set $\underline{E}_{0}^{1}=\underline{J}_{0}^{2}=0$ we get $\underline{U}_{N N_{1}}=0 \Leftrightarrow N \equiv N_{1}$ even if part of the circuit impedances are asymmetric and/or current $\left\{J^{2}\right\}$ and/or voltage $\left\{E^{1}\right\}$ systems are unbalanced (by definition a balanced system has $\underline{\psi}_{0}=\underline{\psi}_{2}=0$ and $\underline{\psi}_{1} \neq 0$ symmetrical components). It may also happen that the impedance bracket of (7) zeroes not for any fundamental reason, but just by sheer numerical witchery and therefore we do not examine it further here.

Additionally, conditions so as to have $\underline{U}_{N N_{2}}=0$ or $\underline{U}_{N_{1} N_{2}}=0$ or both in the Circuit 4 can be worked out along similar lines and will be given in the following section.

\section{Worked examples}

\section{A. Millman's Laboratory}

The first example is a straightforward but involved combinatorial analysis of the so called "Millman's Laboratory" (ML for short), the three phase power circuit shown in Figure 9 whose USPEC equivalent is depicted in Figure 10. This circuit is provided with several "knobs" (or switches) so that $5184=4 \cdot 2^{4} \cdot 3^{4}$ different states are reachable.

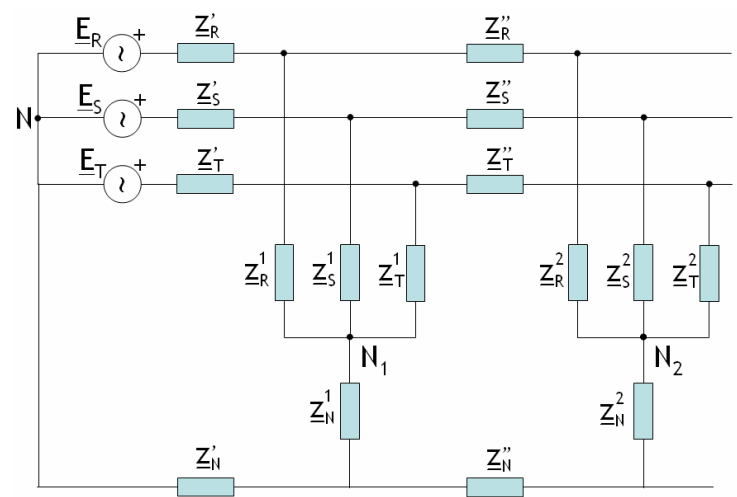

Figure 9: "Millman's Laboratory"

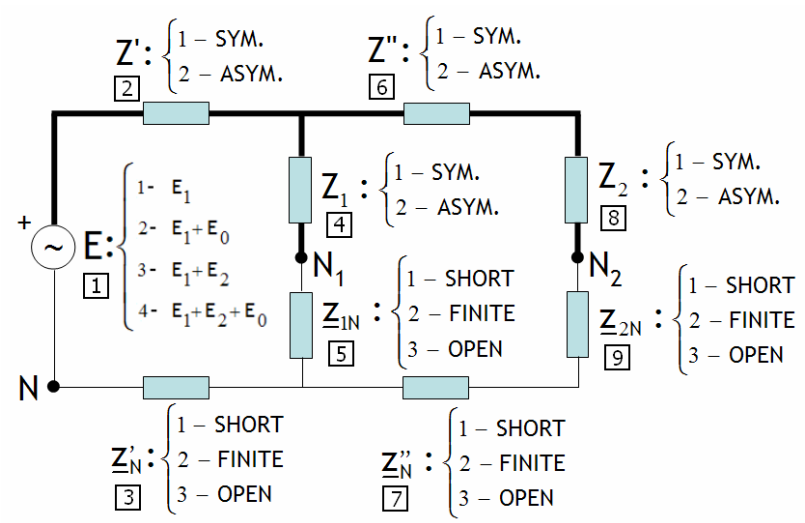

Figure 10: USPEC equivalent of "Millman's Laboratory" with the switch labels indicated

Notice that the voltage source can present different mixtures of positive, negative and zero (homopolar) sequences. In the Appendix we present an Octave script that allows to fully analyse this ML. When this piece of code is run we get the results shown in Figure 11.

All in all we get 984 circuits with $N \equiv N_{1}, 420$ with $N \equiv N_{2}$ and 564 with $N_{1} \equiv N_{2}$. The name of the game is to give a rationale for these numbers, i.e., we want to analyse, classify and count all the circuits that meet these 
specific conditions related to neutral-point potential differences (NPPD).

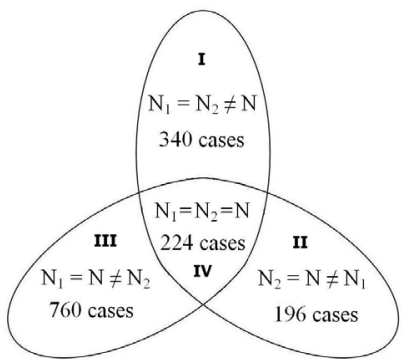

Figure 11: Result of the analysis of Millman's Laboratory

NPPD can be null for three distinct reasons and each case must be dealt with separately. NPPD are zero:

A. when the neutral points are SHORTED.

B. when MNT (in the version presented in section 4) enforces equality among two neutral points only. As shown this is related to the presence of symmetric impedances and the absence of certain homopolar currents and voltages in part of the circuit. We refer to this case as 2MNT as well.

C. when condition B applies to the whole circuit so that all of the neutral points become electrically equal. As regards Millman's Laboratory, we have obviously that $N=N_{1} \& N=N_{2} \Rightarrow N=N_{1}=N_{2}$. We can refer to this case also as FMNT (for full-MNT).

To begin with we can very easily account for Case IV because it is a well known fact that when the voltage sources are "balanced" and all the impedances are symmetric we get the full neutral point equality $N=N_{1}=N_{2}$. See Table 2 .

Table 2: switch state, multiplicity and sum totals for Case IV

\begin{tabular}{|c|c|c|c|c|c|c|c|c|c|c|}
\hline switch & $i_{1}$ & $i_{2}$ & $i_{3}$ & $i_{4}$ & $i_{5}$ & $i_{6}$ & $i_{7}$ & $i_{8}$ & $i_{9}$ & Par. \\
\hline $\mathrm{A}$ & $1-4$ & 1,2 & 1 & 1,2 & 1 & 1,2 & 1 & 1,2 & 1 & \\
\hline$\mu$ & 4 & 2 & 1 & 2 & 1 & 2 & 1 & 2 & 1 & 64 \\
\hline $\mathrm{C}$ & 1,3 & 1 & $1-3$ & 1 & $1-3$ & 1 & $1-3$ & 1 & $1-3$ & \\
\hline$\mu$ & 2 & 1 & 3 & 1 & 3 & 1 & 3 & 1 & 3 & 162 \\
\hline $\mathrm{A} \& \mathrm{C}$ & 1,3 & 1 & 1 & 1 & 1 & 1 & 1 & 1 & 1 & \\
\hline$\mu$ & 2 & 1 & 1 & 1 & 1 & 1 & 1 & 1 & 1 & 2 \\
\hline & & & & & & & & \multicolumn{5}{|c|}{ Total } & 224 \\
\hline
\end{tabular}

In this table columns labelled $i_{1}-i_{9}$ are impedance switches. Rows show their state $(i-j$ expressions stand for integer ranges) and below them " $\mu$ rows" give their multiplicity factors. "Par." means "partial sum" and the set theoretical result $|X \cup Y|=|X|+|Y|-|X \cap Y|$ is used to obtain the Total sum.

We move on and next, in order to examine case B above, we have to ascertain under which conditions 2MNT applies. Thus, for each neutral point voltage balance we implement very much the same circuit transformations examined already in Section 4.

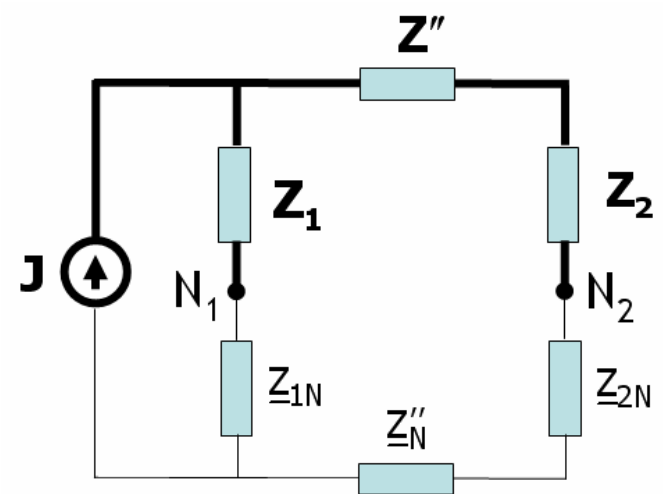

Figure 12: USPEC equivalent used to calculate $\underline{U}_{\mathrm{N} 1 \mathrm{~N} 2}$ (Case I)

Table 3: Correspondences between Circuits 12 and $1(k=$ $\mathrm{R}, \mathrm{S}, \mathrm{T})$

\begin{tabular}{|ccc|}
\hline Figure 12 & $\rightarrow$ & Figure 1 \\
$\underline{z}_{k}^{1} \cdot \underline{J}_{k}$ & $\rightarrow$ & $\underline{E}_{k}$ \\
$-\underline{z}_{N}^{1} \cdot \underline{J}_{0}$ & $\rightarrow$ & $\underline{E}_{N}$ \\
$\underline{z}_{k}^{1}+\underline{z}_{k}^{2}+\underline{z}_{k}^{\prime \prime}$ & $\rightarrow$ & $\underline{z}_{k}$ \\
$\underline{z}_{N}^{1}+\underline{z}_{N}^{2}+\underline{z}_{N}^{\prime \prime}$ & $\rightarrow$ & $\underline{z}_{N}$ \\
\hline
\end{tabular}

Case I $\left(N \neq N_{1}=N_{2}\right)$

Figure 12 shows the suitable reduced equivalent and Table 3 the transformation rules. Now, taking these into account in equation (1) and putting $A \equiv N_{1}$ and $B \equiv N_{2}$ we get in general (save for numerical coincidences):

$$
U_{N_{1} N_{2}}=0 \Leftrightarrow\left\{\begin{array}{c}
\left\{z^{1}, z^{\prime \prime}, z^{2}\right\}-S Y M \\
J_{0}=0
\end{array}\right.
$$

In this case voltage sources impose no condition at all. Neutral return current $\underline{J}_{N}=\underline{J}_{0}$ is zero when either $i_{3}=3$ (open) or $i_{5}=i_{7}=3$ or $i_{5}=i_{9}=3$. This situation is summarised in Figure 13 where out of the 81 possible combinations of switches 3, 5, 7 and 9, exactly 37 make $\underline{J}_{N}=0$ (see the Appendix).

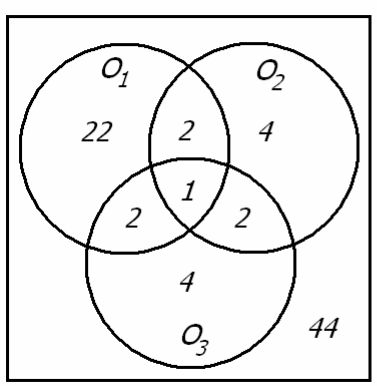

\begin{tabular}{|l|l|l|l|}
\hline & $O_{1}$ & $O_{2}$ & $O_{3}$ \\
\hline$i_{3}$ & 3 & $x$ & $x$ \\
\hline$i_{5}$ & $x$ & 3 & 3 \\
\hline$i_{7}$ & $x$ & 3 & $x$ \\
\hline$i_{9}$ & $x$ & $x$ & 3 \\
\hline
\end{tabular}

Figure 13: $\{3,5,7,9\}$ switch sets such that $\mathrm{J}_{\mathrm{N}}=0$ (Case I) 
Table 4: switch state, multiplicity and sum totals for Case I

\begin{tabular}{|c|c|c|c|c|c|c|c|c|c|c|}
\hline switch & $i_{1}$ & $i_{2}$ & $i_{3}$ & $i_{4}$ & $i_{5}$ & $i_{6}$ & $i_{7}$ & $i_{8}$ & $i_{9}$ & Par. \\
\hline $\mathrm{A}$ & $1-4$ & 1,2 & 2,3 & 1,2 & 1 & 1,2 & 1 & 1,2 & 1 & \\
\hline$\mu$ & 4 & 2 & 2 & 2 & 1 & 2 & 1 & 2 & 1 & 128 \\
\hline $\mathrm{A} \& \mathrm{C}$ & 1,3 & 1 & 2,3 & 1 & 1 & 1 & 1 & 1 & 1 & \\
\hline$\mu$ & 2 & 1 & 2 & 1 & 1 & 1 & 1 & 1 & 1 & -4 \\
\hline $\mathrm{A}^{*}=\mathrm{A}-\mathrm{C}$ & & & & & & & & & & 124 \\
\hline $\mathrm{B}$ & $1-4$ & 1,2 & $\begin{array}{c}1-3 \\
(\mathrm{a})\end{array}$ & 1 & $\begin{array}{c}1-3 \\
(\mathrm{a})\end{array}$ & 1 & $\begin{array}{c}1-3 \\
(\mathrm{a})\end{array}$ & 1 & $\begin{array}{c}1-3 \\
(\mathrm{a})\end{array}$ & \\
\hline$\mu$ & 4 & 2 & 37 & 1 & & 1 & & 1 & & 296 \\
\hline $\mathrm{B} \& \mathrm{C}$ & 1,3 & 1 & $1-3$ & 1 & $1-3$ & 1 & $1-3$ & 1 & $1-3$ & \\
\hline$\mu$ & 2 & 1 & 37 & 1 & & 1 & & 1 & & -74 \\
\hline $\mathrm{B}^{*}=\mathrm{B}-\mathrm{C}$ & & & & & & & & & & 222 \\
\hline $\mathrm{A} \& \mathrm{~B}$ & $1-4$ & 1,2 & 3 & 1 & 1 & 1 & 1 & 1 & 1 & \\
\hline$\mu$ & 4 & 2 & 1 & 1 & 1 & 1 & 1 & 1 & 1 & 8 \\
\hline $\mathrm{A} \& \mathrm{~B} \& \mathrm{C}^{\prime}$ & 1,3 & 1 & 1 & 1 & 1 & 1 & 1 & 1 & 1 & \\
\hline$\mu$ & 2 & 1 & 1 & 1 & 1 & 1 & 1 & 1 & 1 & -2 \\
\hline $\mathrm{A}^{*} \& \mathrm{~B}^{*}$ & & & & & & & & & & 6 \\
\hline & & & & & & & & \\
\hline
\end{tabular}

Table 4 shows the detailed combinatorial analysis for Case I. Note (a) means that these switch states are restricted as indicated in Fig. 13. As a rule we work out first the equalities (e.g. $N_{1}=N_{2}$ ) by the running criterion (e.g. A). Then we calculate the subset already in Case IV (e.g. A \& C ), which must be subtracted (e.g. $\left.\mathrm{A}^{*}=\mathrm{A}-\mathrm{C}=\mathrm{A} \cap \overline{\mathrm{C}}\right)$. Notice that the set theory equation $A^{*} \& B^{*}=A \& B-C$ has been used.

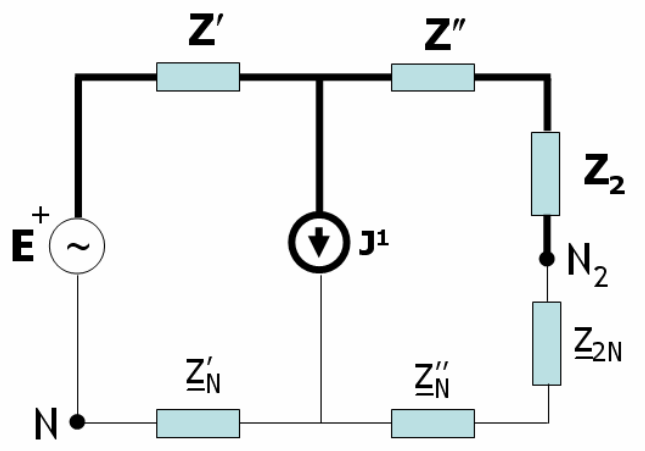

Figure 14: USPEC equivalent used to calculate $\underline{\mathrm{U}}_{\mathrm{N} 2 \mathrm{~N}}$ (Case II)

Table 5: Correspondences between Circuits 14 and $1(k=$ $\mathrm{R}, \mathrm{S}, \mathrm{T})$

\begin{tabular}{|ccc|}
\hline Figure 14 & $\rightarrow$ & Figure 1 \\
$\underline{E}_{k}+\left(\underline{z}_{k}^{2}+\underline{z}_{k}^{\prime \prime}\right) \cdot \underline{J}_{k}^{1}$ & $\rightarrow$ & $\underline{E}_{k}$ \\
$-\left(\underline{z}_{N}^{2}+\underline{z}_{N}^{\prime \prime}\right) \cdot \underline{J}_{0}^{1}$ & $\rightarrow$ & $\underline{E}_{N}$ \\
$\underline{z}_{k}^{\prime}+\underline{z}_{k}^{2}+\underline{z}_{k}^{\prime}$ & $\rightarrow$ & $\underline{z}_{k}$ \\
$\underline{z}_{N}^{\prime}+\underline{z}_{N}^{2}+\underline{z}_{N}^{\prime \prime}$ & $\rightarrow$ & $\underline{z}_{N}$ \\
\hline
\end{tabular}

Case II $\left(N=N_{2} \neq N_{1}\right)$

Figure 14 shows the suitable reduced equivalent and Table 5 the transformation rules. Taking these into account in equation (1) and setting $A \equiv N_{2}$ and $B \equiv N$ we get as a rule that, except for punctual numerical coincidences:

$$
U_{N_{2} N}=0 \Leftrightarrow\left\{\begin{array}{c}
\left\{z^{\prime}, z^{\prime \prime}, z^{2}\right\}-S Y M \\
\underline{J}_{0}^{1}=\underline{E}_{0}=0
\end{array}\right.
$$

Noticeably unbalanced but non homopolar voltage sources are allowed, that is, voltage systems such that their phase-to-phase voltages close triangles whose barycentres lie at the origin of the complex plane. Obviously the neutral return current $\underline{J}_{N}^{1}=\underline{J}_{0}^{1}$ is zero for these switch combinations: either $i_{5}=3$ or $i_{3}=i_{7}=3$ or $i_{3}=i_{9}=3$. This calculation mimics that of Case I examined before and on display in Figure 13 with the result that again 37 out of 81 combinations of switches 3, 5, 7 and 9 nullify $J_{N}^{1}$. The detailed combinatorial analysis for Case II is displayed in Table 6 below.

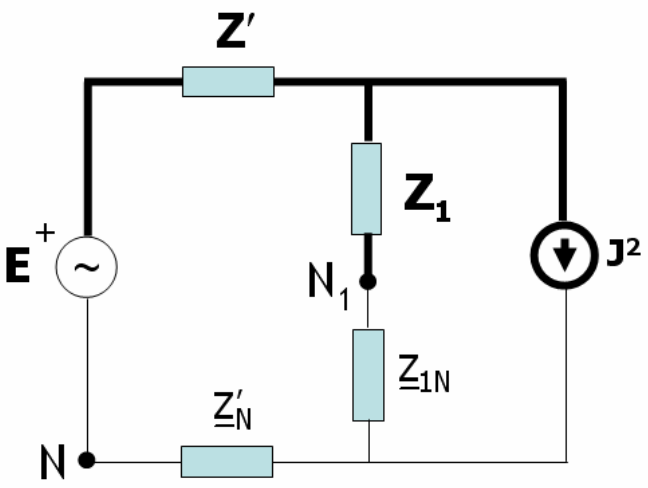

Figure 15: USPEC equivalent used to analyse $\underline{\mathrm{U}}_{\mathrm{N} 1 \mathrm{~N}}$ (Case III)

Table 6: switch state, multiplicity and sum totals for Case II

\begin{tabular}{|c|c|c|c|c|c|c|c|c|c|c|}
\hline switch & $i_{1}$ & $i_{2}$ & $i_{3}$ & $i_{4}$ & $i_{5}$ & $i_{6}$ & $i_{7}$ & $i_{8}$ & $i_{9}$ & Par. \\
\hline A & $1-4$ & 1,2 & 1 & 1,2 & 2,3 & 1,2 & 1 & 1,2 & 1 & \\
\hline$\mu$ & 4 & 2 & 1 & 2 & 2 & 2 & 1 & 2 & 1 & 128 \\
\hline $\mathrm{A} \& \mathrm{C}$ & 1,3 & 1 & 1 & 1 & 2,3 & 1 & 1 & 1 & 1 & \\
\hline$\mu$ & 2 & 1 & 1 & 1 & 2 & 1 & 1 & 1 & 1 & -4 \\
\hline $\mathrm{A}^{*}=\mathrm{A}-\mathrm{C}$ & & & & & & & & & & 124 \\
\hline B & 1,3 & 1 & $\begin{array}{l}1-3 \\
\text { (a) }\end{array}$ & 1,2 & $\begin{array}{l}1-3 \\
\text { (a) }\end{array}$ & 1 & $\begin{array}{l}1-3 \\
\text { (a) }\end{array}$ & 1 & $\begin{array}{l}1-3 \\
\text { (a) }\end{array}$ & \\
\hline$\mu$ & 2 & 1 & 37 & 2 & & & & & & 148 \\
\hline$B \& C$ & 1,3 & 1 & $\begin{array}{l}1-3 \\
\text { (a) }\end{array}$ & 1 & $\begin{array}{l}1-3 \\
\text { (a) }\end{array}$ & 1 & $\begin{array}{l}1-3 \\
\text { (a) }\end{array}$ & 1 & $\begin{array}{l}1-3 \\
\text { (a) }\end{array}$ & \\
\hline$\mu$ & 2 & 1 & 37 & 1 & & 1 & & 1 & & -74 \\
\hline $\mathrm{B}^{*}=\mathrm{B}-\mathrm{C}$ & & & & & & & & & & 74 \\
\hline$A \& B$ & 1,3 & 1 & 1 & 1,2 & 3 & 1 & 1 & 1 & 1 & \\
\hline$\mu$ & 2 & 1 & 1 & 2 & 1 & 1 & 1 & 1 & 1 & 4 \\
\hline $\mathrm{A} \& \mathrm{~B} \& \mathrm{C}$ & 1,3 & 1 & 1 & 1 & 1 & 1 & 1 & 1 & 1 & \\
\hline$\mu$ & 2 & 1 & 1 & 1 & 1 & 1 & 1 & 1 & 1 & -2 \\
\hline$A^{*} \& B^{*}$ & & & & & & & & & & 2 \\
\hline & & & & & & & & & al & 196 \\
\hline
\end{tabular}

Table 7: Correspondences between Circuits 15 and $1(k=$ $\mathrm{R}, \mathrm{S}, \mathrm{T})$

\begin{tabular}{|ccc|}
\hline Figure 15 & $\rightarrow$ & Figure 1 \\
$\underline{E}_{k}+\underline{z}_{k}^{1} \cdot \underline{J}_{k}^{2}$ & $\rightarrow$ & $\underline{E}_{k}$ \\
$-\underline{z}_{N}^{1} \cdot \underline{J}_{0}^{2}$ & $\rightarrow$ & $\underline{E}_{N}$ \\
$\underline{z}_{k}^{1}+\underline{z}_{k}^{\prime}$ & $\rightarrow$ & $\underline{z}_{k}$ \\
$\underline{z}_{N}^{1}+\underline{z}_{N}^{\prime}$ & $\rightarrow$ & $\underline{z}_{N}$ \\
\hline
\end{tabular}

Case III $\left(N=N_{1} \neq N_{2}\right)$

Figure 15 shows the proper reduced equivalent and Table 7 the transformation rules. Taking these into account in equation (1) and setting $A \equiv N$ and $B \equiv N_{1}$ we get in general (numerical conspiracies apart):

$$
U_{N_{1} N}=0 \Leftrightarrow\left\{\begin{array}{c}
\left\{z^{\prime}, z^{1}\right\}-S Y M \\
\underline{J}_{0}^{2}=\underline{E}_{0}=0
\end{array}\right.
$$


It is apparent that the neutral return current $\underline{J}_{N}^{2}=\underline{J}_{0}^{2}$ is zero when either $i_{7}=3$ or $i_{9}=3$ or $i_{3}=i_{5}=3$. This situation is summarised in Figure 16 where out of the 81 possible combinations of switches 3, 5, 7 and 9, exactly 49 make $J_{0}^{2}=0$ (see the Appendix). The detailed combinatorial analysis for Case III is shown in Table 8 below. Notes: (a) case $i_{7}=i_{9}=1$ is excluded; (b) conforms to results stated in Fig: 16 ; (c) $i_{7}=3 \mid i_{9}=3$ (inclusive OR).

Hereby the analysis of ML has been successfully completed.

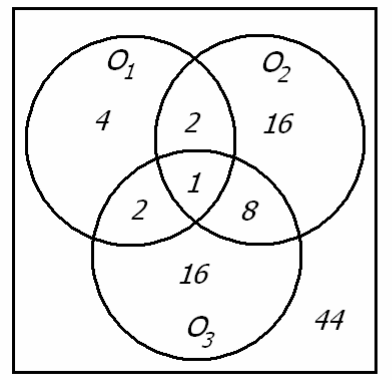

\begin{tabular}{|c|c|c|c|}
\hline & $O_{1}$ & $O_{2}$ & $O_{3}$ \\
\hline$i_{3}$ & 3 & $x$ & $x$ \\
\hline$i_{5}$ & 3 & $x$ & $x$ \\
\hline$i_{7}$ & $x$ & 3 & $x$ \\
\hline$i_{9}$ & $x$ & $x$ & 3 \\
\hline
\end{tabular}

Figure 16: $\{3,5,7,9\}$ switch sets such that $\underline{J}_{0}^{2}=0$ (Case III)

Table 8: switch state, multiplicity and sum totals for Case III

\begin{tabular}{|c|c|c|c|c|c|c|c|c|c|c|}
\hline switch & $i_{1}$ & $i_{2}$ & $i_{3}$ & $i_{4}$ & $i_{5}$ & $i_{6}$ & $i_{7}$ & $i_{8}$ & $i_{9}$ & Par. \\
\hline A & $1-4$ & 1,2 & 1 & 1,2 & 1 & 1,2 & $\begin{array}{l}1-3 \\
\text { (a) }\end{array}$ & 1,2 & $\begin{array}{l}1-3 \\
\text { (a) }\end{array}$ & \\
\hline$\mu$ & 4 & 2 & 1 & 2 & 1 & 2 & 8 & 2 & & 512 \\
\hline $\mathrm{A} \& \mathrm{C}$ & 1,3 & 1 & 1 & 1 & 1 & 1 & $\begin{array}{l}1-3 \\
\text { (a) }\end{array}$ & 1 & $\begin{array}{l}1-3 \\
\text { (a) }\end{array}$ & \\
\hline$\mu$ & 2 & 1 & 1 & 1 & 1 & 1 & 8 & 1 & & -16 \\
\hline $\mathrm{A}^{*}=\mathrm{A}-\mathrm{C}$ & & & & & & & & & & 496 \\
\hline B & 1,3 & 1 & $\begin{array}{l}1-3 \\
\text { (b) }\end{array}$ & 1 & $\begin{array}{l}1-3 \\
\text { (b) }\end{array}$ & 1,2 & $\begin{array}{l}1-3 \\
\text { (b) }\end{array}$ & 1,2 & $\begin{array}{l}1-3 \\
\text { (b) }\end{array}$ & \\
\hline$\mu$ & 2 & 1 & 49 & 1 & & 2 & & 2 & & 392 \\
\hline $\mathrm{B} \& \mathrm{C}$ & 1,3 & 1 & $\begin{array}{l}1-3 \\
\text { (b) }\end{array}$ & 1 & $\begin{array}{l}1-3 \\
\text { (b) }\end{array}$ & 1 & $\begin{array}{l}1-3 \\
\text { (b) }\end{array}$ & 1 & $\begin{array}{l}1-3 \\
\text { (b) }\end{array}$ & \\
\hline$\mu$ & 2 & 1 & 49 & 1 & & 1 & & 1 & & -98 \\
\hline $\mathrm{B}^{*}=\mathrm{B}-\mathrm{C}$ & & & & & & & & & & 294 \\
\hline$\overline{A \& B}$ & $\overline{1,3}$ & 1 & 1 & 1 & 1 & 1,2 & (c) & 1,2 & (c) & \\
\hline$\mu$ & 2 & 1 & 1 & 1 & 1 & 2 & 5 & 2 & & 40 \\
\hline $\mathrm{A} \& \mathrm{~B} \& \mathrm{C}$ & 1,3 & 1 & 1 & 1 & 1 & 1 & (c) & 1 & (c) & \\
\hline$\mu$ & 2 & 1 & 1 & 1 & 1 & 1 & 5 & 1 & & -10 \\
\hline$A^{*} \& B^{*}$ & & & & & & & & & & 30 \\
\hline & & & & & & & & \multicolumn{2}{|c|}{ Total } & 760 \\
\hline
\end{tabular}

\section{B. The single-phase equivalent circuit}

The second example revolves around the analysis of the same circuit examined in subsection $5 \mathrm{~A}$, but the point to be made is so fundamental that it is worth a whole subsection by itself. In fact, it concerns the immediate application of our restated MNT to the derivation of the all-important single-phase equivalent circuit (SPEC) of a symmetrically loaded three phase circuit fed with a balanced voltage system. In the last subsection we started the analysis of ML in a somewhat cavalier manner, hurrying to get rid of Case IV in the first place. Now, however well known any fact may be, it still deserves a proof!. As it stands, the derivation of the SPEC in a number of electrical engineering textbooks [3] is not logically satisfactory due to this same oversight. So, whereas standard MNT does not directly apply to
Circuit 9, our revised version indeed does. We must proceed by imposing pairwise the electrical equality of neutral points (2MNT) until these have been exhausted and the FMNT nirvana reached. For instance, in ML it is only after we combine (any two of) equations (8), (9) and (10) that we get a satisfactory proof of this "well known fact".

FMNT is the cornerstone result from which SPEC readily follows: the equipotentiality of all the neutrals entails the decoupling of the phases. This situation can be vividly illustrated (see Figure 17) by physically connecting the neutrals (altogether inconsequential) which effectively short-circuits the remaining phases.

\section{Conclusions}

Millman's Network Theorem is standard fare for every electrical engineer. In this work we have given a simple but nice proof thereof; next we have presented MNT in a matrix setting and applied it specifically to three phase power circuits, and finally we have enlarged its application domain by considering more complex geometries. Some examples have been thoroughly discussed and commented computer codes given away.

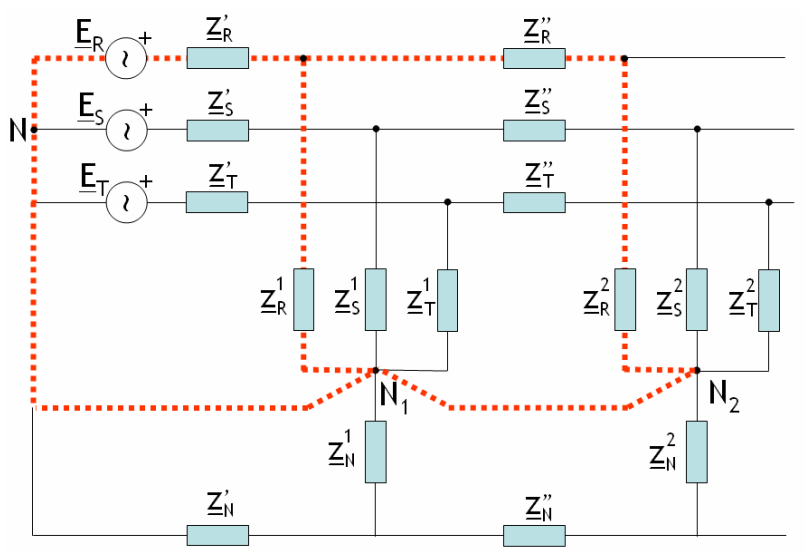

Figure 17: Graphical proof of SPEC when the FMNT condition applies to Circuit 9

\section{Appendix}

As already mentioned, in order to get a double check on these results, we have extensively used short scripts written in the Octave/Matlab computer language that have helped us to numerically analyse this set of circuits. Attached to this document we provide these fully functional Octave scripts, which naturally have been implemented using the USPEC method. Now, the simplest Matlab/Octave implementation of the USPEC method requires the following constants and anonymous functions:

Table 9: simplest USPEC implementation in Octave language

\begin{tabular}{|l|l|}
\hline$(1)$ & $\mathrm{a}=\exp \left(\mathrm{j}^{*} 2 / 3^{*} \mathrm{pi}\right) ;$ \\
\hline$(2)$ & $\mathrm{big}=1 \mathrm{e} 6 ;$ \\
\hline$(3)$ & $\mathrm{doz}=@(\mathrm{a}, \mathrm{b})\left(\mathrm{b}^{*} \operatorname{ones}(3)+\operatorname{diag}(\mathrm{a})\right) ;$ \\
\hline$(4)$ & $\mathrm{d} 2 \mathrm{y}=@(\mathrm{a}, \mathrm{b}, \mathrm{c})\left(\left[\mathrm{a}^{*} \mathrm{c}, \mathrm{b}^{*} \mathrm{a}, \mathrm{c}^{*} \mathrm{~b}\right] . /(\mathrm{a}+\mathrm{b}+\mathrm{c})\right) ;$ \\
\hline$(5)$ & $\mathrm{zpz}=@(\mathrm{a}, \mathrm{b})\left(\mathrm{a}^{*} \operatorname{inv}(\mathrm{a}+\mathrm{b}) * \mathrm{~b}\right) ;$ \\
\hline
\end{tabular}


An explanation of Table 9 is in order:

(1) is the three-phase "operator".

(2) is a "big enough" number to represent an open circuit; by the same token $1 /$ big represents a short circuit.

(3) Implements equation (3) and the calling syntax is Zmatrix $=\operatorname{doz}\left(\left[\underline{\mathbf{z}}_{1}, \underline{\mathrm{Z}}_{2}, \underline{\mathrm{z}}_{3}\right], \underline{\mathrm{z}}_{\mathrm{n}}\right)$.

(4) Implements Kennelly's delta-to-star transformation. The calling syntax is $\left[\underline{\mathrm{z}}_{1}, \underline{\mathrm{z}}_{2}, \underline{\mathrm{z}}_{3}\right]=\mathrm{d} 2 \mathrm{y}\left(\underline{\mathrm{z}}_{12}, \underline{\mathrm{Z}}_{23}, \underline{\mathrm{Z}}_{31}\right)$ so that the matrix impedance of a delta connected load writes: Zdelta $=\operatorname{doz}\left(\mathrm{d} 2 \mathrm{y}\left(\underline{\mathrm{z}}_{12}, \underline{\mathrm{Z}}_{23}, \underline{\mathrm{Z}}_{31}\right)\right.$, big $)$.

(5) $Z_{12}=\mathrm{zpz}\left(\mathrm{Z}_{1}, \mathrm{Z}_{2}\right)$ gives the parallel association of matrix impedances $Z_{1}$ and $Z_{2}$, and is the matrix counterpart of the scalar rule $\underline{\mathbf{z}}_{12}=\underline{\mathbf{z}}_{1} * \underline{\mathbf{z}}_{2} /\left(\underline{\mathbf{z}}_{1}+\underline{\mathbf{z}}_{2}\right)$. Obviously series association of impedances goes as: $\mathrm{Z}_{12}=\mathrm{Z}_{1}+\mathrm{Z}_{2}$

(6) Calculates the measurement of a wattmeter which sees $\mathrm{U}$ and I three-phase systems and is connected between phases a, b (voltmeter) and c (ammeter).

The list of the scripts included in the paper is given in Table 10 below.

Table 10: list of the provided Octave scripts (m files)

\begin{tabular}{|l|l|}
\hline$(1)$ & MNT_example_1.m \\
\hline$(2)$ & MNT_example_2.m \\
\hline$(3)$ & INULL_switch_state_case_I.m \\
\hline$(4)$ & INULL_switch_state_case_II.m \\
\hline$(5)$ & INULL_switch_state_case_III.m \\
\hline$(6)$ & millmans_laboratory.m \\
\hline$(7)$ & lab_millman_case_analysis.m \\
\hline
\end{tabular}

Here we briefly describe what these scripts are used for:

(1) This script uses standard USPEC (see Table 9) to calculate a numerical example of Circuit 4.

(2) This script illustrates the recursive use of USPEC as explained in the main text.

(3) (4) and (5) serve to calculate the multiplicity of the null current conditions as reported in Figures 13 and 16.

(6) This piece of code calculates the 5184 different occurrences of ML and stores the results in a file.

(7) This script reads the file outputted by the script (6) and analyses the data case by case in order to produce the results stated in Figure 10 and in the rightmost column of Tables 2, 4, 6 and 8 .

\section{References}

[1] M. A. Laughton, D. J. Warne, "Electrical Engineer's Reference Book", $16^{\text {th }}$ edition, Newnes, 2003

[2] http://en.wikipedia.org/wiki/Millman\%27s theorem

[3] J. Fraile Mora, "Electromagnetismo y Circuitos Eléctricos", 4 ed., McGraw Hill, 2005

[4] G. Aguirre-Zamalloa, F. Uriondo-Arrue, J. R. Hernández-González, "Classroom Analysis of ThreePhase Circuits with the Universal Single Phase Equivalent Circuit", Proceedings of XCLEEE, 2007 and references therein

[5] http://www.gnu.org/software/octave/

[6] http://en.wikipedia.org/wiki/Modified nodal analysis

[7] http://en.wikipedia.org/wiki/Block matrix

[8] http://en.wikipedia.org/wiki/Mathematical induction

[9] http://en.wikipedia.org/wiki/Th\%C3\%A9venin\%27s theorem

[10] http://en.wikipedia.org/wiki/Symmetrical components 